\title{
EFEITO DA UTILIZAÇÃO DE DENTIFRÍCIOS COM DIFERENTES COMPOSTOS BIOATIVOS NAS PROPRIEDADES SUPERFICIAIS DO ESMALTE DENTAL CLAREADO
}

\section{EFFECT OF TOOTHPASTES WITH DIFFERENT BIOACTIVE COMPOUNDS ON DENTAL BLEACHED ENAMEL SUPERFICIAL PROPERTIES}

\author{
Thayne Waleska Yamamoto* \\ Rubens Corte Real de Carvalho**
}

\begin{abstract}
RESUMO
Este estudo tem como objetivo realizar uma revisão da literatura para verificar se os novos compostos bioativos presentes no mercado, CPP-ACP, fosfossilicato de cálcio e sódio e o nitrato de potássio são eficazes na remineralização do esmalte dental clareado. As alterações da superfície dental ocasionadas pelo clareamento são diversas. Duas novas técnicas de análise demonstram-se promissoras: o QLF e a nanoidentação. O QLF relaciona-se com a autofluorescência dental, possuindo vantagens relacionadas à sua especificidade, simples manuseio e preservação do espécime. Já a nanoidentação é capaz de mensurar a dureza em sítios específicos, em uma escala nanométrica, permitindo uma criteriosa seleção da área a ser analisada. O estudo concluiu que a literatura diverge quanto aos resultados dos efeitos do clareamento na superfície dental e os estudos que avaliaram os diversos compostos bioativos apresentam diferentes metodologias que não permitem o estabelecimento de um protocolo de aplicação dos mesmos. Sendo assim, faz-se necessária a realização de novos estudos para avaliar os possíveis efeitos dos diferentes compostos quando da utilização destes como dentifrícios de uso terapêutico após o tratamento clareador.
\end{abstract}

DESCRITORES: Clareamento dental.

\section{ABSTRACT}

This study aims to review the literature and verify if the new bioactive compounds, like CPP-ACP, calcium sodium phosphosilicate and potassium nitrate are effective on the remineralization of the bleached dental enamel. Two new analysis have shown to be promising: the QLF and the nanoindentation. The QLF is related with the auto fluorescence of the teeth and has advantages related to its specificity, simple manipulation and it is not a destructive technique. The nanoindentation is capable to measure the surface hardness in very specific sites, enabling a criterious selection of the area which will be analyzed.. It was conclusive that the literature disagrees of the results presented about the effects of bleaching on the enamel surface and the researches that evaluated the bioactive compounds have demonstrated differences in their methodologies. These inconclusive findings interfere on the establishment of an application protocol to those new dentifrices. Thus, it is necessary to develop new researches to better understand the possible effects of the bioactive compounds when these dentifrices are related to a therapeutic use after treatment with bleaching agents.

DESCRIPTORS: Tooth Bleaching.

* Mestranda em Dentística. Departamento de Dentística. Faculdade de Odontologia da Universidade de São Paulo - FOUSP. São Paulo - SP.

** Professor titular do Departamento de Dentística. Faculdade de Odontologia da Universidade de São Paulo - FOUSP. São Paulo-SP. 


\section{N T RO DUÇÃO}

O atual conceito de estética incentiva uma busca individual para a adequação dos padrões de beleza estabelecidos. Dessa forma, a estética na Odontologia valorizou-se e o clareamento dental tornou-se um dos procedimentos mais procurados nos consultórios odontológicos pelos pacientes.

O peróxido de hidrogênio $(\mathrm{PH})$ é o ingrediente ativo dos géis clareadores: é um agente oxidante que possui a capacidade de se difundir pelas estruturas dentais mineralizadas em função da permeabilidade desses substratos e devido ao baixo peso molecular dessas substâncias. No entanto, o contato direto dos agentes clareadores com as superfícies dentais mineralizadas por um longo período pode causar efeitos adversos a essas superfícies.

Vários métodos têm sido utilizados na avaliação de modificações ocorridas devido ao clareamento dental, incluindo-se testes que avaliam quantitativamente alterações das propriedades físicas e químicas, como o teste de dureza superficial e a quantificação mineral por meio da fluorescência do elemento dental.

Muitos autores avaliaram a utilização de fluoretos, demonstrando haver efeitos benéficos durante o tratamento de clareamento dental promovendo proteção à estrutura dental devido à reposição dos íons perdidos durante o processo desmineralizador. O mesmo ocorre quando há a incorporação de diversos compostos bioativos nos dentifrícios. De acordo com o mecanismo de ação desses compostos bioativos, a deposição dos íons de diferentes formas pode auxiliar na remineralização e diminuir a desmineralização.

Porém, poucos estudos avaliaram essas características e os resultados ainda são contraditórios; o suposto efeito de aceleração no processo de remineralização desses compostos bioativos sobre o esmalte dental clareado ainda não foi estabelecido efetivamente.

Dentre as novas metodologias de análise, a quantificação mineral por meio da fluorescência do elemento dental (QLFTM) está sendo utilizada para avaliar a superfície de esmalte dental quando do manchamento da estrutura e possíveis alterações subsuperficiais do substrato. Já a mensuração da dureza dental em uma escala nanométrica possibilita uma avaliação em diferentes regiões do esmalte dental. Esse método é caracterizado pela identação em uma área estreita, através de cargas reduzidas, quando comparado com a microidentação.

\section{REVISÃO DA LITERATURA E DISCUSSÃO}

O uso do peróxido de carbamida (PC) a 10\% foi publicado em 1989 por Haywood e Heymann ${ }^{1}$, que descreveram a técnica de clareamento caseiro que não necessitava do uso de um pré-tratamento da superfície com ácido fosfórico ou emprego de qualquer outro ácido para a atuação do gel clareador.

As soluções de PC são muito instáveis e dissociam-se facilmente ao entrarem em contato com o tecido dental ou saliva, formando PH e ureia. Posteriormente, o PH se degradará em oxigênio e água e a ureia em amônia e dióxido de carbono ${ }^{2}$.

É de comum conhecimento que o $\mathrm{PH}$ é o principal agente ativo das reações que possibilitam o clareamento dos dentes. $\mathrm{O}$ seu baixo peso molecular permite que ele se difunda livremente através das estruturas do esmalte e dentina em função da permeabilidade dessas estruturas ${ }^{2,3}$, atuando na formação de radicais livres que irão agir nas cadeias longas das moléculas cromóforas, de coloração escura, degradando-as em moléculas menores, menos pigmentadas e mais difusíveis ${ }^{2,4,5}$. O PH, ao entrar em contato com a superfície dental, se decompõe em água e oxigênio nascente, sua forma reativa, que penetra por entre os prismas de esmalte e alcança a dentina, causa uma quebra das moléculas orgânicas ${ }^{5-7}$.

Dos efeitos adversos clínicos do tratamento clareador os mais relatados pelos pacientes são a sensibilidade dentinária e a irritação gengival ${ }^{8}$. Contudo, o clareamento também pode resultar em alterações estruturais das superfícies dentais ${ }^{9}$. Estudos demonstraram uma influência negativa dos agentes clareadores na integridade das estruturas orgânicas do esmalte ${ }^{10}$. Entretanto, não há relatos de caso ou estudos clínicos que demonstrem danos macroscópicos e clínicos causados pelos
YAMAMOTO TW

CARVALHO RCR

EFEITO DA UTILIZAÇÃO DE DENTIFRÍCIOS

COM DIFERENTES COMPOSTOS

BIOATIVOS NAS

PROPRIEDADES

SUPERFICIAIS DO

ESMALTE DENTAL

CLAREADO

$\therefore 155$

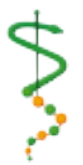

REV, ODONTOL.

UNIV, CID, SÃO

PAULO

2013; 25(2): $154-$

63, MAIO-AGO 
YAMAMOTO TW

CARVALHO RCR

EFEITO DA

UTILIZAÇÃO DE

DENTIFRÍCIOS

COM DIFERENTES

COMPOSTOS

BIOATIVOS NAS

PROPRIEDADES

SUPERFICIAIS DO

ESMALTE DENTAL

CLAREADO

\section{6}

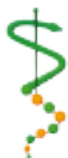

REV, ODONTOL.

UNIV, CID, SÃO

PAULO

$2013 ; 25(2): 154-$

63, MAIO-AGO procedimentos clareadores ${ }^{11}$.

Borges et al. ${ }^{12}$ (2010) relatam que os efeitos do clareamento na microdureza provavelmente estão relacionados ao $\mathrm{pH}$ do gel e este está diretamente envolvido nas alterações da matriz orgânica do esmalte, que ocorrem devido à ação química do PH. Seu efeito oxidante caracteriza uma das principais modificações estruturais ocasionadas pelo clareamento dental, podendo ser intensificado pelo baixo $\mathrm{pH}$ de alguns agentes clareadores, causando diferenças na composição mineral e diminuindo a dureza do esmalte e dentina ${ }^{10,}$ 12,13. Já a desmineralização e perda de íons cálcio são alterações que ocorrem na composição inorgânica do tecido mineralizado, podendo, por vezes, serem passíveis de remineralização ${ }^{14-16}$.

A fim de contornar a sensibilidade dentinária ocasionada pelos géis clareadores, a adição de agentes dessensibilizantes nos produtos clareadores tem sido relatada. Há controvérsias quanto aos reais benefícios ocasionados por essa alteração na composição; porém, estudos clínicos demonstram que o uso de produtos que contêm esses componentes em sua formulação é capaz de diminuir a sensibilidade pós-operatória sem diminuir a eficácia do clareamento ${ }^{8,17-19}$. Basting et al. ${ }^{20}$ (2003) utilizaram um gel de PC $20 \%$ com $3 \%$ de nitrato de potássio e $0.11 \%$ de íons de fluoretos que apresentou as menores diferenças nos valores de microdureza. O mesmo foi observado por Cavalli et al. ${ }^{21}$ (2010) que avaliaram a ação dos clareadores caseiros adicionados de flúor ou cálcio. Contrariamente, Oliveira et al.22(2005) não encontraram efeitos positivos quando da adição de cálcio e fluoretos em diferentes concentrações ao PC a $10 \%$ durante a análise de microdureza superficial da estrutura.

É comumente aceito que os benefícios do flúor se relacionam com a sua capacidade de incorporar-se ao conteúdo mineral na forma de fluorapatita ou hidroxiapatita enriquecida com flúor $^{23}$. Seu uso após o clareamento pode ser indicado a fim de minimizar os possíveis efeitos adversos causados pelos peróxidos nas superfícies dentais mineralizadas e tem demonstrado resultados positivos, possibilitando a pro- teção da estrutura dental devido à reposição dos íons perdidos durante os processos desmineralizadores ${ }^{22,24-26}$. De acordo com uma revisão sistemática realizada por Attin et al..$^{11}(2009)$, o uso de compostos fluoretados concomitantemente ao tratamento clareador pode auxiliar no restabelecimento dos valores de microdureza. $\mathrm{O}$ mesmo autor ${ }^{27}$ (2006) avaliou a influência do peróxido de carbamida na captação de flúor pelo esmalte dental. Os resultados desse estudo indicaram que a fluoretação do esmalte dental clareado é melhor realizada pela aplicação de um gel fluoretado após o tratamento clareador do que um peróxido adicionado de fluoretos.

Wiegand et al..$^{28}$ (2007) demonstraram que o uso de dentifrícios fluoretados após a ação do PC a $10 \%$ podem evitar a diminuição da microdureza. Foi utilizado PC a $10 \%$ durante oito horas por dia, durante 14 dias. Os grupos foram distribuídos entre os diferentes regimes de aplicação de flúor. Concluiu-se que a aplicação regular de dentifrícios fluoretados é suficiente para evitar a perda da microdureza durante o tratamento clareador.

Uma vez que o processo químico da erosão dental também é caracterizado pela desmineralização do substrato dental, é possível objetivar experimentos que verifiquem os efeitos desses compostos bioativos no tecido dental mineralizado submetido ao tratamento clareador. Entre eles estão os dentifrícios que contêm compostos como o fosfopeptídeo de caseína e fosfato de cálcio amorfo (CPP-ACP) (Al Mulahi et al. ${ }^{29}, 2010$, Wegehaupt et al. ${ }^{30}$, 2010), vidro biativo de fosfosilicato de sódio e cálcio (NovaMinß) (Burkwell et al. ${ }^{31}, 2009$, Wefell $\left.{ }^{32}, 2009\right)$, nitrato de potássio e dióxido de titânio (41).

O fosfopeptídeo de caseína e fosfato de cálcio amorfo ou CPP-ACP tem sido utilizado em estudos in vitro e in situ para a remineralização das lesões subsuperficiais do esmalte (Giniger et al. ${ }^{18}$, 2005, Khoroushi et al. $\left.{ }^{33}, 2011\right)$, sendo caracterizado por diminuir o processo de desmineralização da estrutura dental e aumentar a remineralização. Esse processo ocorre porque a caseína pode se ajustar em ambientes ácidos. Num meio com $\mathrm{pH}$ baixo, o ACP se separa do CPP e aumenta os níveis de 
cálcio e fosfato biodisponíveis, mantendo um meio supersaturado no qual pode-se diminuir a desmineralização e promover a remineralização do esmalte através da deposição de apatita ${ }^{13,33,34,35}$.

As soluções desse composto também têm apresentado efeitos na remineralização de lesões subsuperficiais no esmalte. Atualmente muitos estudos avaliam o mecanismo de ação do CPP-ACP nos processos erosivos de forma preventiva ou terapêutica. Poggio et al. ${ }^{36}$ (2002) elaboraram um experimento com três grupos, dos quais dois receberam tratamentos isolados (imersão em refrigerante ou tratamento com agente remineralizante, sem escovação) e o outro a combinação de ambos os tratamentos e concluíram que a aplicação dos dentifrícios contendo CPP-ACP é eficaz na prevenção da erosão dental causada por refrigerantes.

Rees et al. ${ }^{37}$ (2007) realizaram um estudo para comparar dois produtos que são indicados para a prevenção da erosão: um dentifrício que possui CPP-ACP e outro com altos níveis de flúor biodisponível e nitrato de potássio. Após a aplicação única desses dentifrícios os espécimes foram submetidos a um desafio erosivo por 1 hora. Nas comparações estatísticas realizadas, os dois dentifrícios foram capazes de prevenir a erosão dental, não havendo diferença estatística entre os dois grupos.

Abreu et al. ${ }^{5}$ (2011) avaliaram a influência do CPP-ACP incorporados no peróxido de hidrogênio a $7,5 \%$ e 9,5\% na microdureza superficial do esmalte e verificou-se a diminuição dos valores de microdureza em todos os grupos. Os efeitos benéficos que o ACP poderia apresentar só foram verificados nos agentes clareadores de baixas concentrações quando associados com a saliva artificial.

Borges et al. ${ }^{34}$ (2011) modificaram a técnica de autoaplicação através da mistura de um dentifrício contendo CPP-ACP com o gel clareador. Para isso, foi realizado um manchamento prévio dos elementos dentais com o intuito de verificar a eficácia do tratamento clareador modificado. Após o manchamento, os espécimes foram submetidos à aplicação de géis clareadores da forma convencional e de soluções preparadas com os géis e o dentifrício. Os resultados obtidos demonstraram que a microdureza superficial foi aumentada no período pós-tratamento e a modificação da técnica não prejudicou a eficácia do tratamento clareador.

O fosfosilicato de cálcio e sódio, cujo nome comercial é NovaMin $\AA$, é um vidro bioativo que tem sido utilizado nos cuidados da saúde bucal para o tratamento da hipersensibilidade dentinária, através da oclusão física dos túbulos dentinários ${ }^{32}$. Estudos recentes demonstram o potencial desse material de prevenir a desmineralização e auxiliar na remineralização da superfície dental ${ }^{31,38,39}$.

O mecanismo de ação resulta da interação com o meio aquoso e consequente liberação de íons de cálcio, sódio e fosfato: os íons de sódio das partículas do NovaMin ${ }^{\circledR}$ trocam-se rapidamente com os cátions de hidrogênio. Essa troca iônica permite que o cálcio e o fosfato sejam liberados da estrutura da partícula ${ }^{39}$.

Gjorgievska e Nicholson ${ }^{38}$ (2011) objetivaram determinar os efeitos do clareamento na estrutura do esmalte dental e o potencial do vidro bioativo NovaMin ${ }^{\circ}$, presente em dois dentifrícios, para remineralizar as regiões do esmalte dental clareado. Utilizou-se o PC a $16 \%$ aplicado à superfície de terceiros molares humanos, durante 8 minutos, por 7 dias. Dentre os dentifrícios utilizados nos tratamentos um continha $5,5 \%$ do vidro bioativo e o outro $7 \%$. A aplicação dos agentes remineralizadores se deu após o clareamento, pelo mesmo intervalo de tempo. Os espécimes foram analisados qualitativamente através da microscopia eletrônica de varredura (MEV) e quantitativamente pela detecção de energia dispersiva dos raios-x (EDX) e demonstraram que o agente clareador pode causar alterações superficiais morfológicas, porém um tratamento remineralizador pode reparar as mesmas. Houve a formação de uma camada de vidro bioativo e um aumento no conteúdo de cálcio e fosfato no esmalte, assemelhando-se ao substrato não clareado. Dos dentifrícios avaliados, o que continha $7 \%$ de NovaMin ${ }^{\circ}$ apresentou melhores resultados.

Shimaoka $^{39}$ (2011) avaliou o uso dos compostos CPP-ACP e NovaMin $\AA$, associados ou não ao flúor, após diferentes
YAMAMOTO TW

CARVALHO RCR

EFEITO DA UTILIZAÇÃO DE DENTIFRÍCIOS

COM DIFERENTES COMPOSTOS BIOATIVOS NAS PROPRIEDADES SUPERFICIAIS DO ESMALTE DENTAL CLAREADO

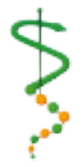

REV, ODONTOL.

UNIV, CID, SÃO PAULO

2013; 25(2): $154-$ 63, MAIO-AGo 
YAMAMOTO TW:

CARVALHO RCR

EFEITO DA

UTILIZAÇÃO DE

DENTIFRÍCIOS

COM DIFERENTES

COMPOSTOS

BIOATIVOS NAS

PROPRIEDADES

SUPERFICIAIS DO

ESMALTE DENTAL

$C L A R E A D O$

\section{$158 \cdots$}

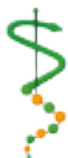

REV, ODONTOL

UNIV, CID, SÃO

PAULO

$2013 ; 25(2): 154-$

63, MAIO-AGO graus de severidade de desafios ácidos. De acordo com os resultados obtidos pela autora, nenhum dos tratamentos remineralizadores propostos foi capaz de reverter totalmente os valores da análise. Entretanto, o tratamento que apresentou maior potencial remineralizador no esmalte dental foi o CPP-ACP adicionado de flúor.

A ação do nitrato de potássio tem sido estudada para o tratamento da sensibilidade dentinária ocasionada pelos tratamentos clareadores. Na presença de um estímulo específico, ocorre a despolarização das fibras sensoriais nervosas nos túbulos dentinários. A consequente repolarização causa a sintomatologia dolorosa. O nitrato de potássio atua prevenindo essa repolarização, reduzindo a excitabilidade das fibras nervosas e também diminuindo a capacidade do nervo de transmitir dor ${ }^{40,41}$. Contudo, estudos relatam que agentes dessensibilizantes com nitrato de potássio possuem ação na obliteração dos túbulos dentinários. Ao avaliar um gel dessensibilizante contendo $2 \%$ de nitrato de potássio e $2 \%$ de fluoreto de sódio, Pinto et al. ${ }^{42}$ (2010) verificaram, através da análise visual por microscopia eletrônica de varredura, a oclusão parcial e total dos túbulos. Os autores também utilizaram a EDX para determinar quais elementos químicos foram depositados próximos aos túbulos e encontraram altos níveis de sódio, magnésio, sílica e potássio. Oberg et al.43 (2009) obtiveram resultados semelhantes ao observarem o uso de géis com $5 \%$ e $10 \%$ de nitrato de potássio.

Kato et al. ${ }^{44}$ (2009) utlizaram dentifrícios à base de nitrato de potássio em seu estudo para avaliar o poder preventivo deste com relação à erosão e verificar se o uso isolado deste e do flúor são mais eficazes do que a combinação de ambos. Dentre os dentifrícios utilizados, um possui fluoreto de sódio e nitrato de potássio a $5 \%$ em sua composição, e dois foram dentifrícios experimentais, também à base de nitrato de potássio a 5\% e o mesmo componente adicionado de flúor. Para a análise foi usado um perfilômetro que mensurou a profundidade erodida. O dentifrício disponível no mercado não demonstrou diferenças estatisticamente significantes quando comparado com o grupo-contro- le. Dos dentifrícios experimentais, o que possuía somente nitrato de potássio na formulação foi capaz de reduzir a erosão. Já a combinação de flúor com o composto dessensibilizante não apresentou o mesmo resultado.

Tay et al. ${ }^{45}$ (2009) verificaram clinicamente que o uso de um gel dessensibilizante contendo $5 \%$ de nitrato de potássio e $2 \%$ de fluoreto de sódio, antes do procedimento clareador, pode diminuir a incidência e a intensidade da sensibilidade sem diminuir a eficácia do clareamento de consultório, utilizando peróxido de hidrogênio a $5 \%$. Outro estudo (Reis et al. ${ }^{40}$, 2011) avaliou a atuação do nitrato de potássio antes do tratamento clareador acelerado por luz, e os resultados foram semelhantes aos obtidos pelos pesquisadores mencionados.

Muitas metodologias necessitam de um polimento inicial dos espécimes culminando na remoção da camada aprismática do esmalte que é hipermineralizada, contém fluorapatita e é mais resistente aos processos desmineralizadores ${ }^{46}$.

A quantificação mineral por meio da fluorescência do elemento dental (QLFTM) foi inicialmente desenvolvida para estudos relacionados com a detecção de lesões incipientes de cárie através de métodos ópticos ${ }^{47}$. O efeito de espalhamento no surgimento de manchas brancas observado pela fluorescência induzida por luz é aumentado pela natureza fluorescente da dentina também pela junção amelodentinária. A presença dessas estruturas abaixo do esmalte é necessária para o fornecimento de contraste suficiente da fluorescência entre esmalte sadio e esmalte lesionado. A estrutura dental autofluoresce quando é incidida por um comprimento de luz específico. Quando há um processo desmineralizador, essa fluorescência é menor do que na estrutura sadia e são apresentadas áreas escurecidas pelo aplicativo do QLFTM ${ }^{47}$.

Estudos recentes demonstram que, pelo uso do QLFTM para a avaliação da superfície dental após o tratamento clareador, é possível comparar-se áreas da estrutura dental clareada e não clareada da mesma maneira que a estrutura desmineralizada e sadia. Pretty et al. ${ }^{47}(2001)$ avaliaram o 
uso do QLF para quantificar a remoção de manchas. Os espécimes utilizados foram armazenados em recipientes imersos num preparado contendo saliva, enxaguatório bucal à base de clorexidina e chá, para se poder estabelecer o manchamento dos corpos de prova. Utilizaram um dentifrício comum para o controle positivo, água destilada como o controle negativo e um dentifrício clareador, ainda em fase de testes. A remoção do manchamento pode ser melhor avaliada pelo QLF devido à sua capacidade de registrar a redução das manchas, longitudinalmente, após os testes. Além disso, o aplicativo do QLF permite a visualização da involução das manchas e a quantificação da ação dos produtos em análise.

Andrade $^{48}$ (2009) realizou um monitoramento in situ do tratamento clareador. Para a avaliação da perda mineral em diferentes momentos foi utilizado o QLF. Foi utilizado um gel clareador de aplicação de consultório e dois autoadministráveis, um contendo ACP em sua composição e outro não. Para essa análise, foram utilizados os valores de $\Delta Q$ fornecidos pelo equipamento. Os agentes clareadores com diferentes composições químicas causaram diferentes níveis de desmineralização no substrato dental e o composto ACP presente em um dos géis foi capaz de reduzir a perda mineral durante o tratamento clareador.

Adeyemi et al..$^{49}$ (2010) realizaram um estudo no qual compararam a técnica da quantificação da fluorescência por luz induzida com o uso do espectrofotômetro para a avaliação do manchamento e clareamento da superfície dental. O pré-tratamento consistia no manchamento da superfície dental e no tratamento clareador dos mesmos espécimes. As duas análises foram registradas e os autores verificaram que há uma alta correlação entre as duas técnicas.

As estruturas dentais mineralizadas são compostas por cristais de cálcio e fosfato, hidroxiapatita primária, proteínas e água em diferentes concentrações. As propriedades mecânicas podem variar em função das taxas desses componentes e da sua composição estrutural ${ }^{50}$.

A nanoidentação tornou-se uma técni- ca conhecida para a realização de testes mecânicos em tecidos duros ${ }^{51-56}$. É caracterizada pela identação em uma área muito estreita, possibilitando um maior número de marcações num mesmo espécime e a precisa seleção da área a ser analisada ${ }^{53}$. Trata-se de um método que pode ser representativo quando o material analisado é homogêneo ou específico de uma região discretamente alterada, quando não homogêneo ${ }^{52}$.

Poucos estudos utilizaram a nanoidentação como método de análise após o clareamento dental; entretanto, há evidências de que a exposição dental a agentes químicos pode causar alterações nas propriedades nanomecânicas do substrato ${ }^{51}$. Zimmerman et al. ${ }^{50}$ (2010) submeteram os espécimes de seu estudo a tratamentos clareadores com a PC $20 \%$ e PH a $10 \%$. Os espécimes que foram tratados com $\mathrm{PH}$ a $10 \%$ demonstraram uma diminuição do módulo de elasticidade do esmalte, enquanto a aplicação de PC a $20 \%$ não apresentou efeitos significantes na superfície dental. Azer et al. ${ }^{53}$ (2008) utilizaram géis clareadores à base de PC a 22\% e PH a $9 \%$ e tiras adesivas autoadministráveis à base de $\mathrm{PH}$ a $10 \%$ e $14 \%$ como agente ativo. As mensurações do módulo de elasticidade e nanodureza demonstraram que houve uma diminuição em todos os grupos nas duas variáveis.

Ushigome et al. ${ }^{57}$ (2009) relatam em seu estudo que muitas pesquisas utilizaram a microdureza Knoop ou Vickers para a avaliação da superfície dental clareada. Porém esses testes necessitam de uma carga aplicada mais elevada e de uma largura de $20-40 \mu \mathrm{m}$ para que as mensurações possam ser realizadas. No sistema de nanoidentação, além de se propiciar o uso de uma carga reduzida, 200mgf, a identação realizada é menor que $1 \mu \mathrm{m}$ e também permite uma análise das alterações subsuperficiais. Para esse estudo, os autores utilizaram o PC a $10 \%$ e $30 \%$. Apesar das concentrações serem diferentes, uma diminuição da dureza foi observada em $20 \mu \mathrm{m}$ abaixo da camada mais superficial do esmalte, mas nenhuma alteração foi verificada a $50 \mu \mathrm{m}$. Pesquisas relacionadas à nanoidentação do substrato dental clareado submetido a diferentes
YAMAMOTO TW

CARVALHO RCR

EFEITO DA UTILIZAÇÃO DE DENTIFRÍCIOS COM DIFERENTES COMPOSTOS BIOATIVOS NAS PROPRIEDADES SUPERFICIAIS DO ESMALTE DENTAL CLAREADO

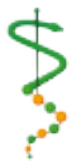

REV, ODONTOL. UNIV. CID, SÃo PAULO

2013; 25(2): $154-$ 63, MAIO-AGO 
YAMAMOTO TW

CARVALHO RCR

EFEITO DA

UTILIZAÇÃO DE

DENTIFRÍCIOS

COM DIFERENTES

COMPOSTOS

BIOATIVOS NAS

PROPRIEDADES

SUPERFICIAIS DO

ESMALTE DENTAL

CLAREADO tratamentos remineralizadores ainda não foram realizadas. Essa metodologia, quando combinada com outras técnicas, pode explanar os reais efeitos das substâncias remineralizadoras, podendo até estabelecer um protocolo de aplicação das mesmas em diferentes casos.

\section{CONCLUSÃO}

Diferentes metodologias e formas de análise têm sido empregadas para avaliar os efeitos dos agentes clareadores na su- perfície, porém não há como predizer que os mesmos se comportem de uma maneira previsível. Os diversos compostos bioativos têm sido amplamente utilizados na indicação da sensibilidade dentinário e os estudos destes com relação às alterações do clareamento dental são poucos. Dessa forma, faz-se necessário um estudo para avaliar os possíveis efeitos dos diferentes compostos quando da utilização destes como dentifrícios de uso terapêutico após o tratamento clareador.

\section{REFERÊNCIAS}

\section{REFERENNCIAS}

1. Haywood VB, Heymann HO. Nightguard vital bleaching. Quintessence Int 1989 Mar;20(3):173-6.

2. Haywood VB, Heymann HO. Nightguard vital bleaching: how safe is it? Quintessence Int 1991 Jul;22(7):51523.

3. Dahl JE, Pallesen U. Tooth bleaching--a critical review of the biological aspects. Crit Rev Oral Biol Med 2003 14(4):292-304.

4. Kihn PW. Vital tooth whitening. Dent Clin North Am 2007 Apr;51(2):31931 , viii.

5. Abreu DR, Sasaki RT, Amaral FL, Florio FM, Basting RT. Effect of home-use and in-office bleaching agents containing hydrogen peroxide associated with amorphous calcium phosphate on enamel microhardness and surface roughness. J Esthet Restor Dent 2011 Jun;23(3):158-68.

6. Tredwin CJ, Naik S, Lewis NJ, Scully C. Hydrogen peroxide tooth-whitening (bleaching) products: review of adverse effects and safety issues. $\mathrm{Br}$ Dent J 2006 Apr 8;200(7):371-6.
7. de Arruda AM, dos Santos PH, Sundfeld RH, Berger SB, Briso AL. Effect of hydrogen peroxide at $35 \%$ on the morphology of enamel and interference in the de-remineralization process: an in situ study. Oper Dent 2012 Sep-Oct; 37(5):518-25.

8. Grobler SR, Majeed A, Moola MH, Rossouw RJ, van Wyk Kotze T. In vivo Spectrophotometric Assessment of the Tooth Whitening Effectiveness of Nite White 10\% with Amorphous Calcium Phosphate, Potassium Nitrate and Fluoride, Over a 6-month Period. Open Dent J 2011 5(18-23.

9. Navimipour EJ, Kimyai S, Nikazar S, Ghojazadeh M. In vitro evaluation of the effect of delaying toothbrushing with toothpaste on enamel microhardness subsequent to bleaching the teeth with 15\% carbamide peroxide. Oper Dent 2012 Jan-Feb;37(1):87-92.

10. Hegedus C, Bistey T, Flora-Nagy E, Keszthelyi $G$, Jenei $A$. An atomic force microscopy study on the effect of bleaching agents on enamel surface. J Dent 1999 Sep;27(7):509-15.

11. Attin T, Schmidlin PR, Wegehaupt F, Wiegand A. Influence of study design on the impact of bleaching agents on dental enamel microhardness: a review. Dent Mater 2009 Feb;25(2):14357. 
12. Borges AB, Yui KC, D'Avila TC, Takahashi CL, Torres CR, Borges AL. Influence of remineralizing gels on bleached enamel microhardness in different time intervals. Oper Dent 2010 Mar-Apr;35(2):180-6.

13. Pinto CF, Oliveira R, Cavalli V, Giannini $M$. Peroxide bleaching agent effects on enamel surface microhardness, roughness and morphology. Braz Oral Res 2004 Oct-Dec;18(4):306-11.

14. Efeoglu N, Wood D, Efeoglu C. Microcomputerised tomography evaluation of $10 \%$ carbamide peroxide applied to enamel. J Dent 2005 Aug;33(7):561-7.

15. Al-Salehi SK, Wood DJ, Hatton PV. The effect of $24 \mathrm{~h}$ non-stop hydrogen peroxide concentration on bovine enamel and dentine mineral content and microhardness. J Dent 2007 Nov;35(11):845-50.

16. Lee KH, Kim HI, Kim KH, Kwon YH. Mineral loss from bovine enamel by a $30 \%$ hydrogen peroxide solution. $f$ Oral Rehabil 2006 Mar;33(3):229-33.

17. Dawson PF, Sharif MO, Smith AB, Brunton PA. A clinical study comparing the efficacy and sensitivity of home vs combined whitening. Oper Dent 2011 Sep-Oct;36(5):460-6.

18. Giniger M, Macdonald J, Ziemba S, Felix $\mathrm{H}$. The clinical performance of professionally dispensed bleaching gel with added amorphous calcium phosphate. J Am Dent Assoc 2005 Mar;136(3):383-92.

19. Attin T, Kielbassa AM, Schwanenberg $M$, Hellwig E. Effect of fluoride treatment on remineralization of bleached enamel. J Oral Rehabil 1997 Apr;24(4):282-6.

20. Basting RT, Rodrigues AL, Jr., Serra MC. The effects of seven carbamide peroxide bleaching agents on enamel microhardness over time. J Am Dent Assoc 2003 Oct;134(10):1335-42.
21. Cavalli V, Rodrigues LK, Paes-Leme $\mathrm{AF}$, Brancalion ML, Arruda MA, Berger $\mathrm{SB}$, et al. Effects of bleaching agents containing fluoride and calcium on human enamel. Quintessence Int 2010 Sep;41(8):e157-65.

22. de Oliveira R, Paes Leme AF, Giannini $M$. Effect of a carbamide peroxide bleaching gel containing calcium or fluoride on human enamel surface microhardness. Braz Dent J 2005 16(2):103-6.

23. Langhorst SE, O'Donnell JN, Skrtic D. In vitro remineralization of enamel by polymeric amorphous calcium phosphate composite: quantitative microradiographic study. Dent Mater 2009 Jul;25(7):884-91.

24. Martin JM, de Almeida JB, Rosa EA, Soares $\mathrm{P}$, Torno $\mathrm{V}$, Rached $\mathrm{RN}$, et al. Effect of fluoride therapies on the surface roughness of human enamel exposed to bleaching agents. Quintessence Int 2010 Jan;41(1):71-8.

25. Leandro GA, Attia ML, Cavalli V, do Rego MA, Liporoni PC. Effects of $10 \%$ carbamide peroxide treatment and sodium fluoride therapies on human enamel surface microhardness. Gen Dent 2008 May;56(3):274-7.

26. Tschoppe P, Neumann K, Mueller J, Kielbassa AM. Effect of fluoridated bleaching gels on the remineralization of predemineralized bovine enamel in vitro. J Dent 2009 Feb;37(2):156-62.

27. Attin T, Albrecht K, Becker K, Hannig C, Wiegand A. Influence of carbamide peroxide on enamel fluoride uptake. J Dent 2006 Oct;34(9):668-75.

28. Wiegand A, Schreier M, Attin T. Effect of different fluoridation regimes on the microhardness of bleached enamel. Oper Dent 2007 Nov-Dec;32(6):610-5.

29. Al-Mullahi AM, Toumba KJ. Effect of slow-release fluoride devices and casein phosphopeptide/amorphous calcium phosphate nanocomplexes on enamel remineralization in vitro. Caries Res 2010 44(4):364-71.
YAMAMOTO TW

CARVALHO RCR

EFEITO DA UTILIZAÇÃO DE DENTIFRÍCIOS COM DIFERENTES COMPOSTOS BIOATIVOS NAS PROPRIEDADES SUPERFICIAIS DO esmalte DENTAL CLAREADO
REV, ODONTOL,

UNIV, CID, SÃO PAULO 2013; 25(2): $154-$ 63, MAIO-AGO 
YAMAMOTO TW CARVALHO RCR

EFEITO DA

UTILIZAÇÃO DE

DENTIFRÍCIOS

COM DIFERENTES

COMPOSTOS

BIOATIVOS NAS

PROPRIEDADES

SUPERFICIAIS DO

ESMALTE DENTAL CLAREADO

162
30. Wegehaupt FJ, Attin T. The role of fluoride and casein phosphopeptide/ amorphous calcium phosphate in the prevention of erosive/abrasive wear in an in vitro model using hydrochloric acid. Caries Res 2010 44(4):358-63.

31. Burwell AK, Litkowski LJ, Greenspan DC. Calcium sodium phosphosilicate (NovaMin): remineralization potential. Adv Dent Res 2009 21(1):35-9.

32. Wefel JS. NovaMin: likely clinical success. Adv Dent Res 2009 21(1):40-3.

33. Khoroushi M, Mazaheri H, Manoochehri A. Effect of CPP-ACP application on flexural strength of bleached enamel and dentin complex. Oper Dent 2011 Jul-Aug;36(4):372-9.

34. Borges BC, Borges JS, de Melo CD, Pinheiro IV, Santos AJ, Braz R, et al. Efficacy of a novel at-home bleaching technique with carbamide peroxides modified by CPP-ACP and its effect on the microhardness of bleached enamel. Oper Dent 2011 Sep-Oct;36(5):521-8.

35. Panich M, Poolthong S. The effect of casein phosphopeptide-amorphous calcium phosphate and a cola soft drink on in vitro enamel hardness. J Am Dent Assoc 2009 Apr;140(4):45560.

36. Poggio C, Lombardini M, Dagna A, Chiesa M, Bianchi S. Protective effect on enamel demineralization of a CPP-ACP paste: an AFM in vitro study. $J$ Dent 2009 Dec;37(12):949-54.

37. Rees J, Loyn T, Chadwick B. Pronamel and tooth mousse: an initial assessment of erosion prevention in vitro. J Dent 2007 Apr;35(4):355-7.

38. Gjorgievska E, Nicholson JW. Prevention of enamel demineralization after tooth bleaching by bioactive glass incorporated into toothpaste. Aust Dent J 2011 Jun;56(2):193-200.

39. Shimaoka AM. Potencial remineralizador de dentifrícios com compostos bioativos no esmalte dental submetido a desafios erosivos de diferentes severidades [Dissertação]. São Paulo: Universidade de São Paulo; 2011.
40. Reis A, Dalanhol AP, Cunha TS, Kossatz S, Loguercio AD. Assessment of tooth sensitivity using a desensitizer before light-activated bleaching. Oper Dent 2011 Jan-Feb;36(1):12-7.

41. Basting RT, Amaral FL, Franca FM, Florio FM. Clinical comparative study of the effectiveness of and tooth sensitivity to $10 \%$ and $20 \%$ carbamide peroxide home-use and $35 \%$ and $38 \%$ hydrogen peroxide in-office bleaching materials containing desensitizing agents. Oper Dent 2012 Sep-Oct;37(5):464-73.

42. Pinto SC, Pochapski MT, Wambier DS, Pilatti GL, Santos FA. In vitro and in vivo analyses of the effects of desensitizing agents on dentin permeability and dentinal tubule occlusion. J Oral Sci 2010 Mar;52(1):23-32.

43. Oberg C Fau - Pochapski MT, Pochapski Mt Fau - Farago PV, Farago Pv Fau - Granado CJF, Granado Cj Fau - Pilatti GL, Pilatti Gl Fau - Santos FA, Fa S. Evaluation of desensitizing agents on dentin permeability and dentinal tubule. 0363-6771 (Print)):

44. Kato Mt Fau - Lancia M, Lancia M Fau - Sales-Peres SHC, Sales-Peres Sh Fau Buzalaf MAR, Ma B. Preventive effect of commercial desensitizing toothpastes on bovine enamel. 1421-976X (Electronic)):

45. Tay LY, Kose C, Loguercio AD, Reis A. Assessing the effect of a desensitizing agent used before in-office tooth bleaching. J Am Dent Assoc 2009 Oct;140(10):1245-51.

46. Zantner C, Beheim-Schwarzbach N, Neumann K, Kielbassa AM. Surface microhardness of enamel after different home bleaching procedures. Dent Mater 2007 Feb;23(2):243-50.

47. Pretty IA, Edgar WM, Higham SM. The use of QLF to quantify in vitro whitening in a product testing model. $\mathrm{Br}$ Dent J 2001 Nov 24;191(10):566-9.
REV. ODONTOL UNIV, CID, SÃO PAULO $2013 ; 25(2): 154-$ 63, MAIO-AGO 
48. Andrade AP. Monitoramento do processo de desmineralização e remineralização do esmalte dental humano durante e após o clareamento dental [Dissertação]. São Paulo: Universidade de São Paulo; 2009.

49. Adeyemi AA, Jarad FD, de Josselin de Jong E, Pender N, Higham SM. The evaluation of a novel method comparing quantitative light-induced fluorescence (QLF) with spectrophotometry to assess staining and bleaching of teeth. Clin Oral Investig 2010 Feb;14(1):1925.

50.Zimmerman B, Datko L, Cupelli M, Alapati S, Dean D, Kennedy M. Alteration of dentin-enamel mechanical properties due to dental whitening treatments. J Mech Behav Biomed Mater 2010 May;3(4):339-46.

51. Habelitz S, Marshall GW, Jr., Balooch M, Marshall SJ. Nanoindentation and storage of teeth. J Biomech 2002 Jul;35(7):995-8.

52. Hairul Nizam BR, Lim CT, Chng HK, Yap AU. Nanoindentation study of human premolars subjected to bleaching agent. J Biomech 2005 Nov;38(11):2204-11.
53. Azer SS, Machado C, Sanchez E, Rashid R. Effect of home bleaching systems on enamel nanohardness and elastic modulus. J Dent 2009 Mar;37(3):185-90.

54. Cuy JL, Mann AB, Livi KJ, Teaford MF, Weihs TP. Nanoindentation mapping of the mechanical properties of human molar tooth enamel. Arch Oral Biol 2002 Apr;47(4):281-91.

55. Oyen ML. Nanoindentation hardness of mineralized tissues. J Biomech 2006 39(14):2699-702.

56. Huja SS, Beck FM, Thurman DT. Indentation properties of young and old osteons. Calcif Tissue Int 2006 Jun;78(6):392-7.

57. Ushigome T, Takemoto S, Hattori M, Yoshinari M, Kawada E, Oda Y. Influence of peroxide treatment on bovine enamel surface--cross-sectional analysis. Dent Mater J 2009 May;28(3):315-23.

Recebido em 21-12-2012

Aceito em 17-06-2013
YAMAMOTO TW

CARVALHO RCR

EFEITO DA

UTILIZAÇÃO DE

DENTIFRÍCIOS

COM DIFERENTES

COMPOSTOS

BIOATIVOS NAS

PROPRIEDADES

SUPERFICIAIS DO

esmalte DENTAL

CLAREADO

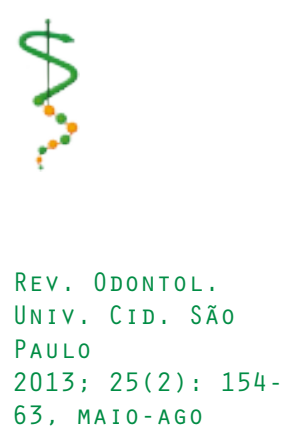

